\title{
Single-finger subcutaneous defibrillation lead and "active can": A novel minimally invasive defibrillation configuration for implantable cardioverter-defibrillator implantation in a young child
}

\author{
Nandini Madan, MD, ${ }^{a}$ J. William Gaynor, MD, ${ }^{\mathrm{b}}$ Ronn Tanel, MD, ${ }^{a}$ Mitchell Cohen, MD, ${ }^{a}$ Susan Nicholson, MD, ${ }^{\mathrm{c}}$ \\ Victoria Vetter, MD, ${ }^{a}$ and Larry Rhodes, MD, ${ }^{a}$ Philadelphia, $\mathrm{Pa}$
}

I mplantable cardioverter defibrillators (ICDs) provide a safe and effective treatment for life-threatening ventricular arrhythmias. Several investigators have demonstrated that ICD use in pediatric patients is feasible, effective, and associated with a low risk of sudden death in follow-up. ${ }^{1}$ However, ICD placement in young children can usually be accomplished only by a thoracotomy approach involving multiple incisions and placement of epicardial defibrillation electrodes. We describe a less invasive yet efficacious lead configuration likely to be associated with a lower complication rate.

\section{Clinical Summary}

A 3.5-year-old, 17.5-kg, previously healthy boy came to the emergency department of a referring hospital in status epilepticus. He was found to be in ventricular fibrillation, which responded to external defibrillation. He was resuscitated and treated with a lidocaine infusion and phenytoin. He made a full neurologic recovery and was transferred to our institution for further management. There was no family history of cardiac disease. His physical examination, electrocardiography, echocardiography, and cardiac magnetic resonance imaging results were normal. A decision was made to implant an ICD for this episode of aborted sudden death. Because of his age, size, and probable lifelong need for defibrillation, the decision was made to place a single subcutaneous defibrillation lead and "active can" configuration.

In the operating room, with the patient under general anesthesia, the apex of the heart was exposed through a subxiphoid incision. A Medtronic 7841 epicardial bipolar pacing lead (Medtronic, Inc, Minneapolis, Minn) was placed on the apex of the right ventricle, and adequate pacing and sensing thresholds were obtained. The Medtronic 6996 T tunneling tool was shaped according to the anatomy of the patient and placed in the introducer sheath, which was then used to create a subcutaneous tunnel. The tunnel was created by blunt dissection at the level of the seventh

From the Divisions of Cardiology, ${ }^{\text {a }}$ Cardiothoracic Surgery, ${ }^{\mathrm{b}}$ and Cardiac Anesthesia, ${ }^{c}$ Children's Hospital of Philadelphia and University of Pennsylvania, Philadelphia, Pa.

Received for publication Oct 29, 2002; revisions requested Jan 6, 2003; revisions received Jan 10, 2003; accepted for publication Feb 11, 2003.

Address for reprints: Nandini Madan, MD, Heart Center for Children, St Christopher's Hospital for Children, Erie Avenue and Front Streets, Philadelphia, PA 19134 (E-mail: nandini.madan@tenethealth.com).

J Thorac Cardiovasc Surg 2003;126:1657-9

Copyright $\odot 2003$ by The American Association for Thoracic Surgery $0022-5223 / 2003 \$ 30.00+0$

doi:10.1016/S0022-5223(03)01032-8

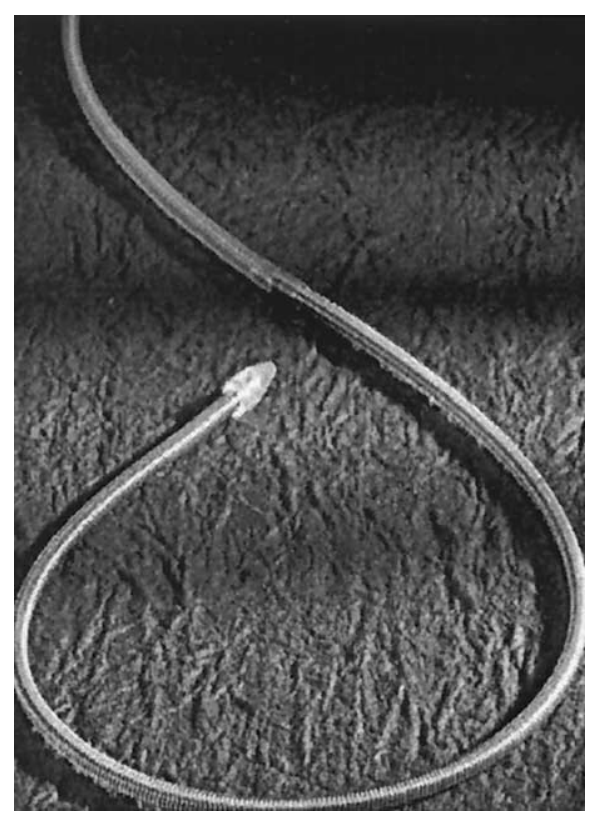

Figure 1. Medtronic model $6996 \mathrm{SQ}$ lead. Note coils of $25-\mathrm{cm}$ long defibrillation electrode at distal tip of lead.

intercostal space and extended posteriorly adjacent to the spine. The creation of the tunnel was guided by the anesthesiologist, directly palpating the lead and anatomic landmarks. This maneuver was used to position the tip of the lead as close to the spine as possible. Previous studies have demonstrated that the most efficient defibrillation vector is achieved by placing the lead between the sixth and tenth intercostal spaces with the tip as close to the spine as possible. ${ }^{2}$ The Medtronic model 6996 SQ lead (Figure 1) was advanced in the sheath. The distal end of the lead has a $25-\mathrm{cm}$ long defibrillation coil with an exposed metal surface area of $5 \mathrm{~cm}^{2}$ and an electrical shadow area of $18 \mathrm{~cm}^{2}$. The introducer sheath was slit open and removed with the stylet and wire. A preperitoneal abdominal pocket was created in the right upper quadrant for a Medtronic Marquis 7274 ICD device. The leads were secured in the appropriate ports and the device was interrogated (Figure 2). After confirmation of adequate sensing, defibrillation threshold (DFT) testing was performed. Ventricular fibrillation was induced with a T-wave shock, and defibrillation was successfully achieved twice with a 16-J biphasic shock. Lower energies were not tested and the device was programmed as a single-zone ventricular fibrillation device programmed to deliver initial therapy of $20 \mathrm{~J}$ and five rescue shocks of $30 \mathrm{~J}$ each. A pericardial drain was placed 

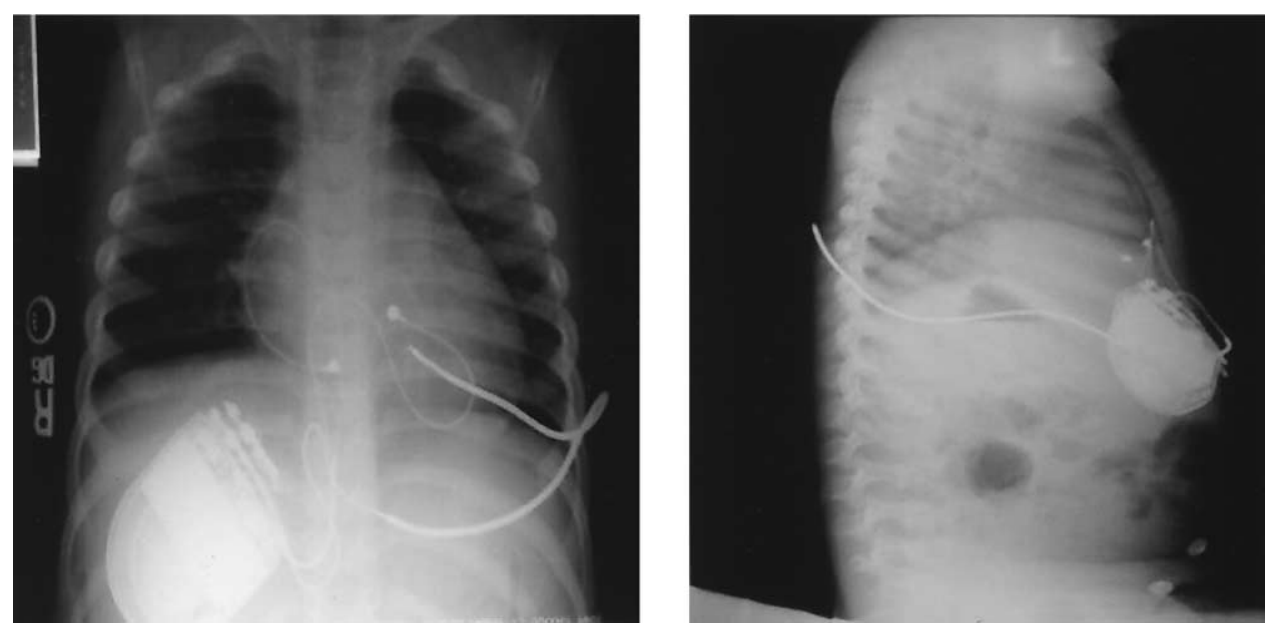

Figure 2. Anteroposterior (A) and lateral (B) chest roentgenograms demonstrating subcutaneous lead in left hemithorax and ICD can in right superior abdominal quadrant. Bipolar epicardial sensing and pacing lead can be seen in anteroposterior projection.

and the incisions were closed. An epidural catheter was placed to provide analgesia. The patient was extubated and transferred to the cardiac intensive care unit. The drain was removed on the first postoperative day. The epidural infusion was discontinued on the second postoperative day. The child was discharged to home on the third postoperative day. He is free of symptoms 5 months after discharge. Repeated DFT testing has been planned for 6 months after implantation.

\section{Discussion}

ICD placement with epicardial leads implanted through a thoracotomy approach has been associated with high incidences of both acute and chronic complications in both pediatric and adult patients. ${ }^{3}$ To overcome these issues, transvenous defibrillation leads, biphasic waveforms, and pectoral implantation of active can devices were developed. ${ }^{4-6}$ However, widespread use of the transvenous ICDs in young patients has been limited by mismatch between patient size and size of defibrillation leads, lack of access to the right ventricle in patients with complex congenital heart disease, and increasing paucity of venous access in patients requiring lifelong device therapy.

The subcutaneous array was developed as an adjunct to a transvenous ICD lead in an effort to provide an increased safety margin in adult patients with high DFTs. Adult studies have demonstrated that the array is at least as effective as an active can or subcutaneous patch in lowering high DFTs. ${ }^{6,7}$ Moreover, subcutaneous arrays have been associated with a lower incidence of side effects than are subcutaneous patches. ${ }^{8}$ There are no reports in the adult literature about the use of the subcutaneous array with an active can without a transvenous defibrillation electrode. However, two recent pediatric case reports and an animal study have documented the successful use of this configuration in children and young animals. ${ }^{9,10}$ With limited follow- up, neither reported any complications of the procedure.

Standard subcutaneous array leads have three fingers and require extensive dissection with creation of three subcutaneous tunnels for placement. However, at least one case series demonstrated that the two-finger array is as efficacious as the triple-finger subcutaneous array configuration in lowering DFTs when used as an adjunct to a transvenous defibrillation electrode. ${ }^{9}$ In another study of adult patients, investigators have demonstrated that elevated DFTs may be significantly decreased with the addition of a single subcutaneous lead to a right ventricular lead active can defibrillation configuration. ${ }^{2}$ The subcutaneous lead is easy to implant, allows flexible lead positioning, and has a long defibrillation coil $(25 \mathrm{~cm})$ with a small lead caliber $(7.5 \mathrm{~F})$. Because of the size of the child, a single subcutaneous lead was used as the anode with an active can as the cathode. This configuration achieved successful defibrillation with low DFTs. This technique, though still requiring placement of an epicardial pacing and sensing lead, requires a single incision that minimizes the surgical approach and may be successfully used in younger and smaller patients with minimal complications. Addition of a second finger to create a subcutaneous array or a patch electrode may be used to lower DFTs as the patient grows. Our case represents use of an innovative minimally invasive technique by which internal defibrillation may be achieved in small and young patients. Additional follow-up is necessary to determine both the stability of the defibrillation thresholds and any long-term complications that maybe associated with this procedure.

\section{References}

1. Silka MJ, Kron J, Dunnigan A, Dick M. Sudden cardiac death and the use of implantable cardioverter-defibrillators in pediatric patients. The Pediatric Electrophysiology Society. Circulation. 1993;87:800-7.

2. Kuhlkamp V, Dornberger V, Khalighi K, Mewis C, Suchalla R, Zeimer G, et al. Effect of single element subcutaneous array electrode added to a transvenous electrode configuration on the defibrillation field and defibrillation threshold. Pacing Clin Electrophysiol. 1998;21: 2596-605.

3. Link MS, Hill SL, Cliff DL, Swygman CA, Foote CB, Homoud MK, et al. Comparison of frequency of complications of implantable car- 
dioverter-defibrillators in children versus adults. Am J Cardiol. 1999; 83:263-6, A5-6.

4. Baker JH, Epstein AE, Voshage-Stahl L. A prospective, randomized evaluation of a nonthoracotomy implantable cardioverter defibrillator lead system. Endotak/PRX Investigator Group. Pacing Clin Electrophysiol. 1997;20:72-8.

5. Bardy GH, Ivey TD, Allen MD, Johnson G, Mehra R, Greene L. A prospective randomized evaluation of biphasic versus monophasic waveform pulses on defibrillation efficacy in humans. $J$ Am Coll Cardiol. 1989;14:728-33.

6. Gradaus MD, Block M, Siedl KH, Brunn J, Isgro F, Hammel D, et al. Defibrillation efficacy comparing a subcutaneous array electrode versus an "active can" implantable cardioverter defibrillator and a subcutaneous array electrode in addition to an "active can" implantable cardioverter defibrillator: results from active can versus array trials I and II. J Cardiovasc Electrophysiol. 2001;12:921-7.
7. Higgins SL, Alexander DC, Kupyers CJ, Brewster SA. The subcutaneous array: a new lead adjunct for the transvenous ICD to lower defibrillation thresholds. Pacing Clin Electrophysiol. 1995;18:1540-8.

8. Kuhlkamp V, Dornberger V, Mewis C, Spiegel L. Comparison of the efficacy of a subcutaneous array electrode with a subcutaneous patch electrode, a prospective randomized study. Int J Cardiol. 2001;78:24756.

9. Gradaus R, Hammel D, Kotthoff S, Bocker D. Nonthoracotomy implantable cardioverter-defibrillator placement in children: use of subcutaneous array leads and abdominally placed implantable cardioverter-defibrillators in children. J Cardiovasc Electrophysiol. 2001;12: 361-2.

10. Berul CI, Triedman JK, Forbess J, Bevilacqua LM, Alexander ME, Dahlby D, et al. Minimally invasive cardioverter defibrillator for children: an animal model and pediatric case report. Pacing Clin Electrophysiol. 2001;24:1789-94. 